\title{
ARTICLE
}

\section{Two-Level Coarse Mesh Finite Difference Formulation with Multigroup Source Expansion Nodal Kernels}

\author{
Joo Il YOON and Han Gyu JOO* \\ Department of Nuclear Engineering, Seoul National University, San 56-1, Sillim-dong, Seoul 151-744, Korea
}

(Received November 30, 2007 and accepted in revised form March 5, 2008)

\begin{abstract}
As an effort to establish a fast, yet accurate multigroup nodal solution method that is crucial in repeated static and transient calculations for advanced reactors, the source expansion form of the semi-analytic nodal method (SANM) is introduced within the framework of the coarse mesh finite difference (CMFD) formulation. The source expansion is to expand the analytic form of the source appearing in the groupwise neutron diffusion equation with a set of orthogonal polynomials in order to obtain a group decoupled analytic solution. Both one- and two-node formulations are examined to determine the best nodal kernel. For the acceleration, a two-level CMFD scheme is established employing a multigroup and two-group CMFD. In addition, an alternative two-node direct SANM formulation with a quartic polynomial is examined to assess the direct vs. iterative resolution of the group coupling. The performance of the CMFD formulation with three different multigroup SANM nodal kernels is examined for a wide variety of multigroup benchmark problems including several MOX-loaded LWR cores and large FBR cores. It is demonstrated that superior accuracy is achievable with all the SANM kernels while the iterative two-node SANM kernel outperforms the others in the multigroup calculations employing more than two groups, and the two-level CMFD formulation is quite efficient in the acceleration of the outer iteration.
\end{abstract}

KEYWORDS: nodal method, CMFD, SANM, multigroup, one-node kernel, two-node kernel

\section{Introduction}

Although the recent trend in the development of the reactor analysis methods is to enhance the solution accuracy by increasing the level of modeling sophistication, there is still a need for an efficient multigroup nodal solver. This is because sophisticated methods such as direct whole core transport calculation methods or simplified $\mathrm{P}_{3}$ pin-by-pin calculation methods are not fast enough to be applied to routine design analyses and to simulations involving repeated transient calculations. This work here is to develop a very fast yet accurate multigroup nodal solver that can be applied to transient as well as steady-state calculations for the advanced LWR (light water reactor) core designs and also for the fast reactors having rectangular fuel assemblies. It was motivated first by the need for a high-fidelity simulator for a transmutation fast reactor, and also by the need for implementing a multigroup capability into an existing LWR nodal kinetics code.

Among the various transverse-integrated nodal methods including the nodal expansion method (NEM), the analytic nodal method (ANM), and the semi-analytic nodal method (SANM), there have been renewed interests in SANM in the last decade because of the ease of multigroup extension while retaining the ANM level of solution accuracy. Origi-

${ }^{*}$ Corresponding author: joohan@snu.ac.kr

(C)Atomic Energy Society of Japan nally, SANM represented the method of Esser and Smith, ${ }^{1)}$ which employs the analytic solution only for the thermal group flux with a polynomial representation of the fast flux in the two-group nodal calculation. Later, Zimin and Ninokata $^{2,3)}$ expanded the notion of SANM such that it represents a nodal method involving a multigroup flux solution given in terms of exponential and polynomial functions. The choice of the exponential functions, specifically in the form of sinh and cosh functions, is dictated by the analytic solution to be obtained after moving all the group source terms to the RHS and then approximating it with a polynomial. Since the analytic solution procedure is applied only partially, the term semi-analytic makes sense. In the actual derivation of SANM by Zimin and Ninokata, they used the same flux form in the source term as well as in the loss terms. Namely, the source terms contain the exponential functions as well. Therefore the SANM of Zimin and Ninokata is here referred to as the flux expansion form of SANM as opposed to the source expansion form to be introduced later. The polynomial part of their basis functions is only up to the second order. They demonstrated that the accuracy of their SANM is essentially the same as that of ANM. ${ }^{2)}$ This method was later applied to a spatial kinetics code, NLSANMT, by Liao and Xie. ${ }^{4)}$

There was a variant of SANM called SANEM (semi-analytic nodal expansion method) developed by Kim et $a .^{5 \text { ) }}$ Kim used a quartic polynomial representation of the source so that the groupwise flux solution consists of a 4th-order 
polynomial particular solution and sinh and cosh homogeneous solutions. In order to determine the source expansion coefficients, Kim used exactly the same method as the one used in the NEM formulation, which uses a node average value, two surface values, and two weighted residual conditions. The first- and second-order polynomials are used in the weighted residual procedure. Kim's SANEM is regarded as the source expansion form of SANM.

On the other hand, $\mathrm{Fu}$ and $\mathrm{Cho}^{6)}$ also worked on the flux expansion form of SANM. The form of the flux expansion of $\mathrm{Fu}$ and Cho is essentially the same as that of Zimin and Ninokata in that they used a quadratic polynomial in the polynomial part. They employed matrix forms of the multigroup neutron diffusion equations and corresponding matrix functions so that the derivation process could be succinct. They proved also that SANM has essentially the same accuracy as ANM.

Compared with the flux expansion form, the source expansion form provides an advantage of decoupling group dependence and the ease of increasing the source expansion order. In order to take this advantage and the generic advantage of SANM, the source expansion form of SANM is employed in this work. Like in Kim's work, ${ }^{5)}$ a quartic expansion of the source is to be used in order to avoid the error associated with a low-order source expansion. However, the source expansion coefficients will be determined differently such that the third and fourth moments of the fluxes can be used to enhance the accuracy of the approximation.

As an acceleration method for the nodal calculation, the coarse mesh finite difference $(\mathrm{CMFD})^{7)}$ formulation is widely used. A local nodal problem solver is necessary in the CMFD formulation and the local problem can be constructed either with a one-node or two-node configuration. In the onenode scheme, the incoming partial currents are specified at the surfaces of a node of interest as the boundary condition, and the one-dimensional flux shape in each direction is determined so that the node average flux and the outgoing partial currents can be newly determined. In the two-node scheme, the node average fluxes of the two nodes are specified as the constraints, and the flux shape satisfying the current and flux continuity at the interface of the two nodes as well as the node average flux constraints are determined. In general, the two-node scheme provides better convergence characteristics than the one-node scheme primarily because better current vs. node average flux relations can be obtainable from the two-node scheme in which the properties of both nodes of an interface are reflected simultaneously in the determination of the interface current. The better convergence characteristic of the two-node scheme was proved analytically through a Fourier analysis. ${ }^{8)}$

There is, however, several favorable aspects in the onenode formulation. First of all, it is simple since it involves only one node of interest and the spatial sweep is to be done for the nodes, not for the surfaces as for the two-node case. But the most important aspect in the view of multigroup calculation is that the multigroup spectrum within a node can be updated using the one-node calculation while it is not possible with the two-node scheme, which uses the average multigroup fluxes as the input parameters. If the node-wise multigroup spectrum is available, it is possible to obtain two-group cross sections from the multigroup cross sections by group collapsing. The subsequent CMFD calculation can then be performed in the two-group structure without performing multigroup CMFD calculations at all. Therefore, the one-node scheme is very easy to implement into an existing two-group code. This was demonstrated by earlier work. $^{9,10)}$ On the contrary, a multigroup CMFD solution is inevitable in the two-node scheme. Since a multigroup (MG) CMFD calculation converges slower than the twogroup (2G) CMFD calculation because of the source iteration, it would be beneficial to establish a $2 \mathrm{G}$ CMFD from the MG CMFD to accelerate the MG calculation. Then, the efficient two-group solution method such as the Wielandt eigenvalue shift method and the simultaneous group solution scheme (as opposed to the groupwise solution scheme) can be utilized. The two-level CMFD formulation in this work is to use two levels of the group structure, namely $2 \mathrm{G}$ and MG.

In the following section, the source expansion form of SANM, which we will refer to as SENM (source expansion nodal method), is derived for both one-node and two-node configurations. Since SENM requires a source iteration, a criterion to check the source shape convergence is introduced as well. The flux expansion form of the two-node SANM is then derived with a quartic polynomial term for a consistent comparison of the direct two-node scheme with an iterative scheme. In Section III, the two-level CMFD calculation scheme is formulated. The performances of the one-node and two-node schemes, and the direct two-node and iterative two-node schemes are assessed in Section IV for a wide range of multigroup eigenvalue problems to determine the best multigroup solution scheme.

\section{Nodal Kernels}

The nodal method considered here is based on the transverse-integrated neutron diffusion equation that can be written as follows in terms of the normalized coordinate variable $\xi_{u}=\frac{2 u}{h_{u}}$, where $h_{u}$ is the node width in the $u$-direction, with the omission of the subscript $u$ for brevity:

$$
\begin{aligned}
- & \frac{4 D_{g}}{h^{2}} \frac{d^{2} \phi_{g}(\xi)}{d \xi^{2}}+\Sigma_{r g} \phi_{g}(\xi) \\
= & \frac{\chi_{g}}{k_{e f f}} \psi(\xi)+S_{g}(\xi)-L_{g}(\xi)
\end{aligned}
$$

with the right-hand-side terms representing the fission source $(\psi)$, scattering source $\left(S_{g}\right)$, and the transverse leakage $\left(L_{g}\right)$. The origin of the coordinate is the center of the node and thus the range of the normalized coordinate is $[-1,1]$. This choice of normalization is dictated by the choice of Legendre polynomial $P_{i}(\xi)$ for the source expansion. It is supposed that the transverse leakage function is given by a quadratic polynomial. In the following, the source expansion form of SANM is first derived for the one-node and twonode configurations, respectively. It is then followed by the derivation of the flux expansion form for the twonode configuration. 


\section{Source Expansion Nodal Method}

In the 4th-order source expansion nodal method (SENM), the fission and scattering sources are approximated using a quartic polynomial so that the entire right-hand side (RHS) of Eq. (1) can be represented as

$$
Q_{g}(\xi) \equiv \frac{\chi_{g}}{k_{e f f}} \psi(\xi)+S_{g}(\xi)-L_{g}(\xi) \cong \sum_{i=0}^{4} q_{g i} P_{i}(\xi) .
$$

Here, it should be noted that the quadratic polynomial for transverse leakage, $L_{g}(\xi)$, is given externally. The quadratic polynomial coefficients can be obtained by requiring the conservation of three node average values of transverse leakage (one from its own node and two from adjacent nodes). The single polynomial of Eq. (2) includes the transverse leakage terms. The specific process to determine the source expansion coefficients $\left(q_{g i}\right)$ will be presented at the end of this section.

Given the RHS as a quartic polynomial, the analytic solution of Eq. (1) is obtained as the following:

$$
\begin{aligned}
\phi_{g}(\xi) & =\phi_{g}^{H}(\xi)+\phi_{g}^{P}(\xi) \\
& =A_{g} \sinh \left(\kappa_{g} \xi\right)+B_{g} \cosh \left(\kappa_{g} \xi\right)+\sum_{i=0}^{4} c_{g i} P_{i}(\xi)
\end{aligned}
$$

where the superscripts $H$ and $P$ stand for the homogeneous and particular solutions, respectively, and $\kappa_{g}=\frac{h}{2} \sqrt{\frac{\Sigma_{r g}}{D_{g}}}$.

The coefficients of the particular solution, $c_{g i}$ 's, are all determined uniquely in terms of the source term coefficients as follows:

$$
\begin{aligned}
& c_{g 0}=\frac{1}{\Sigma_{r g}}\left(q_{g 0}+\frac{1}{\kappa_{g}^{2}}\left(3 q_{g 2}+10 q_{g 4}\right)+\frac{1}{\kappa_{g}^{4}} 105 q_{g 4}\right), \\
& c_{g 1}=\frac{1}{\Sigma_{r g}}\left(q_{g 1}+\frac{15}{\kappa_{g}^{2}} q_{g 3}\right), \\
& c_{g 2}=\frac{1}{\Sigma_{r g}}\left(q_{g 2}+\frac{35}{\kappa_{g}^{2}} q_{g 4}\right), \\
& c_{g 3}=\frac{q_{g 3}}{\Sigma_{r g}}, \text { and } c_{g 4}=\frac{q_{g 4}}{\Sigma_{r g}} .
\end{aligned}
$$

The coefficients of the homogeneous solution should be determined by imposing the constraint or boundary conditions that are applied differently in the one-node and two-node configurations as presented in the following sections.

Suppose that all the homogeneous solution coefficients are determined so that the solution given by Eq. (3) is uniquely determined. Then the flux shape involving the sinh and cosh functions can be approximated using a quartic polynomial given as Eq. (5) using the orthogonal property of Legendre polynomials:

$$
\phi_{g}(\xi) \cong \tilde{\phi}_{g}(\xi) \equiv \bar{\phi}_{g}+\sum_{i=1}^{4} a_{g i} P_{i}(\xi)
$$

where

$$
\begin{aligned}
& a_{g i}=\frac{\left\langle\phi_{g}(\xi), P_{i}(\xi)\right\rangle}{\left\langle P_{i}(\xi), P_{i}(\xi)\right\rangle}, \\
& \bar{\phi}_{g} \equiv a_{g 0}=c_{g 0}+\frac{\sinh \left(\kappa_{g}\right)}{\kappa_{g}} B_{g}, \\
& a_{g 1}=c_{g 1}+\frac{3}{\kappa_{g}}\left(\cosh \left(\kappa_{g}\right)-\frac{\sinh \left(\kappa_{g}\right)}{\kappa_{g}}\right) A_{g}, \\
& a_{g 2}=c_{g 2}-5\left(\frac{3 \cosh \left(\kappa_{g}\right)}{\kappa_{g}^{2}}-\left(1+\frac{3}{\kappa_{g}^{2}}\right) \frac{\sinh \left(\kappa_{g}\right)}{\kappa_{g}}\right) B_{g}, \\
& a_{g 3}=c_{g 3}+\frac{7}{\kappa_{g}}\left(\left(1+\frac{15}{\kappa_{g}^{2}}\right) \cosh \left(\kappa_{g}\right)-\left(6+\frac{15}{\kappa_{g}^{2}}\right) \frac{\sinh \left(\kappa_{g}\right)}{\kappa_{g}}\right) A_{g}, \text { and } \\
& a_{g 4}=c_{g 4}-9\left(\frac{5}{\kappa_{g}^{2}}\left(2+\frac{21}{\kappa_{g}^{2}}\right) \cosh \left(\kappa_{g}\right)-\left(1+\frac{45}{\kappa_{g}^{2}}+\frac{105}{\kappa_{g}^{4}}\right) \frac{\sinh \left(\kappa_{g}\right)}{\kappa_{g}}\right) B_{g} .
\end{aligned}
$$

Here, the bracket stands for the inner product of the functions involving an integral over $[-1,1]$. Note that the orthogonal expansion corresponds to the least-square fitting of a function and it is equivalent to the weighted residual procedure, which reads

$$
\int_{-1}^{1} P_{i}(\xi)\left(\phi_{g}(\xi)-\tilde{\phi}_{g}(\xi)\right) d \xi=0 \quad(i=0 \ldots 4)
$$

and was employed by Lee et al. ${ }^{11)}$ in their derivation of the source expansion form of the two-node SANM kernel. Once the quartic flux expansion is known for each group, the main source term consisting of the fission and scattering sources can be expressed as a fourth-order polynomial as well. The quadratic transverse leakage term is then merely added to the main source term to finalize the source polynomial $Q_{g}(\xi)$.

(1) One-Node Kernel

In the one-node formulation, the incoming partial currents are specified as the boundary conditions at the two surfaces of the node. The two boundary conditions would uniquely define the two homogeneous solution coefficients, $A_{g}$ and $B_{g}$. However, when a multidimensional problem is solved with the one-node configuration, it is desirable to determine these two coefficients considering the multidimensional nodal balance, which means a simultaneous resolution of the transverse-integrated one-dimensional diffusion equations 
of all directions. This simultaneous solution process is detailed below.

The transverse leakage term contains the node average transverse leakage that can be written as follows when the transverse directions are $y$ and $z$ :

$$
l_{g 0}^{x}=\frac{J_{y g}^{r}-J_{y g}^{l}}{h_{y}}+\frac{J_{z g}^{r}-J_{z g}^{l}}{h_{z}}
$$

where the superscripts, $r$ and $l$, designate the right and left surfaces of the node so that $J_{y g}^{r}$ is the $y$-directional surface average current at the right $x-z$ surface of the node. If the simultaneous solution is to be obtained for the node of interest, the average currents of all directions will change together, meaning that the constant transverse leakage term of Eq. (8) cannot be fixed yet. In this situation, we can assume that only the shape defined by the first- and second-order terms in the quadratic transverse leakage polynomial is fixed, but the constant traverse leakage term is yet to be determined.

With this interpretation, the zeroth-order source term, $q_{g 0}$, is considered to be not fixed either, and thus, the constant term of the particular solution, $c_{g 0}$, cannot be determined yet as in Eq. (4). However, it can be represented in terms of the node average flux, which is now considered unknown, and the even term coefficient $B_{g}$ as

$$
c_{g 0}=\bar{\phi}_{g}-\frac{\sinh \left(\kappa_{g}\right)}{\kappa_{g}} B_{g}
$$

by integrating Eq. (3) according to the definition of the node average flux: $\bar{\phi}_{g}=\frac{1}{2} \int_{-1}^{1} \phi(\xi) d \xi$.

The solution can now be rewritten as

$$
\begin{aligned}
\phi_{g}(\xi)= & \bar{\phi}_{g}+A_{g} \sinh \left(\kappa_{g} \xi\right) \\
& +B_{g}\left(\cosh \left(\kappa_{g} \xi\right)-\frac{\sinh \left(\kappa_{g}\right)}{\kappa_{g}}\right)+\phi_{g}^{\tilde{p}}(\xi)
\end{aligned}
$$

where the nonconstant part of the particular solution is defined as

$$
\phi_{g}^{\tilde{p}}(\xi)=\sum_{i=1}^{4} c_{g i} P_{i}(\xi) .
$$

By applying the incoming current boundary condition, $A_{g}$ and $B_{g}$ can be represented in terms of the given incoming currents and the node average flux $\bar{\phi}_{g}$, which is yet to be determined.

The incoming partial currents at the left and right surfaces of the node are given as

$$
\begin{aligned}
J_{g}^{r-} & =\frac{1}{4} \phi_{g}(1)-\frac{1}{2} J_{g}(1) \\
\text { and } J_{g}^{l-} & =\frac{1}{4} \phi_{g}(-1)+\frac{1}{2} J_{g}(-1),
\end{aligned}
$$

where the minus sign in the superscript signifies the negative direction to the outward normal vector of the surface. With the derivative of the normalized variable, the net current is given as

$$
\begin{aligned}
J_{g}(\xi) & =-\frac{2 D_{g}}{h} \frac{d \phi_{g}(\xi)}{d \xi} \\
& =-2 \beta_{g} \kappa_{g}\left(A_{g} \cosh \left(\kappa_{g} \xi\right)+B_{g} \sinh \left(\kappa_{g} \xi\right)\right)+J_{g}^{\tilde{p}}(\xi)
\end{aligned}
$$

where $\beta_{g}=\frac{D_{g}}{h}$ and $J_{g}^{\tilde{p}}(\xi)=-\frac{2 D_{g}}{h} \frac{d \phi_{g}^{\tilde{p}}(\xi)}{d \xi}=-2 \beta_{g} \sum_{i=1}^{4} c_{g i} P_{i}^{\prime}(\xi)$. Now, Eq. (12) can be solved for $A_{g}$ and $B_{g}$ after inserting the flux of Eq. (10) to give

$$
\left[\begin{array}{cc}
\alpha_{g}^{s-} & \alpha_{g}^{c-} \\
-\alpha_{g}^{s-} & \alpha_{g}^{c-}
\end{array}\right]\left[\begin{array}{l}
A_{g} \\
B_{g}
\end{array}\right]=\left[\begin{array}{l}
\gamma_{g}^{r} \\
\gamma_{g}^{l}
\end{array}\right] .
$$

Here,

$$
\begin{aligned}
\alpha_{g}^{s-} & =\beta_{g} \kappa_{g} \cosh \left(\kappa_{g}\right)+\frac{1}{4} \sinh \left(\kappa_{g}\right), \\
\alpha_{g}^{c-} & =\beta_{g} \kappa_{g} \sinh \left(\kappa_{g}\right)+\frac{1}{4}\left(\cosh \left(\kappa_{g}\right)-\frac{\sinh \left(\kappa_{g}\right)}{\kappa_{g}}\right), \\
\gamma_{g}^{r} & =J_{g}^{r-}-\frac{1}{4}\left(\bar{\phi}_{g}+\phi_{g}^{\tilde{p} r}\right)+\frac{1}{2} J_{g}^{\tilde{p} r}, \\
\gamma_{g}^{l} & =J_{g}^{l-}-\frac{1}{4}\left(\bar{\phi}_{g}+\phi_{g}^{\tilde{p} l}\right)-\frac{1}{2} J_{g}^{\tilde{p} l}, \\
\phi_{g}^{\tilde{p} r} & \equiv \phi_{g}^{\tilde{p}}(1)=c_{g 1}+c_{g 2}+c_{g 3}+c_{g 4}, \\
\phi_{g}^{\tilde{p} l} & \equiv \phi_{g}^{\tilde{p}}(-1)=-c_{g 1}+c_{g 2}-c_{g 3}+c_{g 4}, \\
J_{g}^{\tilde{p} r} & \equiv J_{g}^{\tilde{p}}(1)=-2 \beta_{g}\left(c_{g 1}+3 c_{g 2}+6 c_{g 3}+10 c_{g 4}\right), \text { and } \\
J_{g}^{\tilde{p} l} & \equiv J_{g}^{\tilde{p}}(-1)=-2 \beta_{g}\left(c_{g 1}-3 c_{g 2}+6 c_{g 3}-10 c_{g 4}\right) .
\end{aligned}
$$

The homogeneous solution coefficients are then obtained as

$$
\begin{aligned}
A_{g} & =\frac{\gamma_{g}^{r}-\gamma_{g}^{l}}{2 \alpha_{g}^{s-}} \\
& =\frac{2\left(J_{g}^{r-}-J_{g}^{l-}\right)-\left(1+4 \beta_{g}\right) c_{g 1}-\left(1+24 \beta_{g}\right) c_{g 3}}{4 \beta_{g} \kappa_{g} \cosh \left(\kappa_{g}\right)+\sinh \left(\kappa_{g}\right)} \text { and } \\
B_{g} & =\frac{\gamma_{g}^{r}+\gamma_{g}^{l}}{2 \alpha_{g}^{c-}} \\
& =\frac{-\bar{\phi}_{g}+2\left(J_{g}^{r-}+J_{g}^{l-}\right)-\left(1+12 \beta_{g}\right) c_{g 2}-\left(1+40 \beta_{g}\right) c_{g 4}}{4 \beta_{g} \kappa_{g} \sinh \left(\kappa_{g}\right)+\cosh \left(\kappa_{g}\right)-\frac{\sinh \left(\kappa_{g}\right)}{\kappa_{g}}} .
\end{aligned}
$$

Note that $A_{g}$ is determined in terms of the incoming currents, while $B_{g}$ is yet to be determined since it contains the unknown node average flux.

By inserting these homogeneous solution coefficients into Eq. (13), the net current is now obtained as a function of the node average flux and incoming currents. The net current relation is then inserted into the multidimensional nodal balance equation for the one node, which reads

$$
\sum_{u=x, y, z} \frac{J_{g u}(1)-J_{g u}(-1)}{h_{u}}+\Sigma_{r g} \bar{\phi}=\frac{\chi_{g}}{k_{e f f}} \bar{\psi}+\bar{S}_{g} .
$$

The nodal balance equation contains the $u$-directional leakage given below omitting the $u$ index

$$
\begin{aligned}
\Delta \tilde{J}_{g} & =\frac{1}{h}\left(J_{g}(1)-J_{g}(-1)\right) \\
& =-\Sigma_{D g}\left(\kappa_{g} \sinh \left(\kappa_{g}\right) B_{g}+3 c_{g 2}+10 c_{g 4}\right)
\end{aligned}
$$

where the diffusion cross section is defined in this case as

$$
\Sigma_{D g} \equiv \frac{4 \beta_{g}}{h}=\frac{4 D_{g}}{h^{2}} .
$$


This nodal balance equation contains the net currents of all directions that contain the common unknown, $\bar{\phi}_{g}$. It can be solved for the node average flux, which finally gives

$$
\bar{\phi}_{g}=\frac{\bar{s}_{g}+\sum_{u=x, y, z} \Sigma_{D g}^{u}\left(2 \tau_{g}^{u}\left(J_{g u}^{l-}+J_{g u}^{r-}\right)+\left(3-\tau_{g}^{u}\left(1+12 \beta_{g}^{u}\right)\right) c_{g 2}^{u}+\left(10-\tau_{g}^{u}\left(1+40 \beta_{g}^{u}\right)\right) c_{g 4}^{u}\right)}{\Sigma_{r g}+\sum_{u=x, y, z} \Sigma_{D g}^{u} \tau_{g}^{u}}
$$

where $u$ appearing in the superscript or subscript designates the direction and

$$
\begin{aligned}
\tau_{g}^{u} & =\frac{\kappa_{g}^{u} \sinh \left(\kappa_{g}^{u}\right)}{4 \beta_{g}^{u} \kappa_{g}^{u} \sinh \left(\kappa_{g}^{u}\right)+\cosh \left(\kappa_{g}^{u}\right)-\frac{\sinh \left(\kappa_{g}^{u}\right)}{\kappa_{g}^{u}}} \\
\bar{s}_{g} & =\chi_{g} \bar{\psi}^{\prime}+\sum_{g^{\prime}=1}^{G} \Sigma_{g^{\prime} g} \bar{\phi}_{g}^{\prime} .
\end{aligned}
$$

with the prime sign designating the values from the previous iteration step.

Once the node average flux is determined, $B_{g}$ is also determined using Eq. (15) and the outgoing current can be determined as follows:

$$
\begin{aligned}
{\left[\begin{array}{c}
J_{g}^{r+} \\
J_{g}^{l+}
\end{array}\right]=} & {\left[\begin{array}{c}
\frac{1}{4} \phi_{g}(1)+\frac{1}{2} J_{g}(1) \\
\frac{1}{4} \phi_{g}(-1)-\frac{1}{2} J_{g}(-1)
\end{array}\right] } \\
= & {\left[\begin{array}{cc}
\alpha_{g}^{s+} & \alpha_{g}^{c+} \\
-\alpha_{g}^{s+} & \alpha_{g}^{c+}
\end{array}\right]\left[\begin{array}{c}
A_{g} \\
B_{g}
\end{array}\right] } \\
& +\left[\begin{array}{c}
\frac{1}{4}\left(\bar{\phi}_{g}+\phi_{g}^{\tilde{p} r}\right)+\frac{1}{2} J_{g}^{\tilde{p} r} \\
\frac{1}{4}\left(\bar{\phi}_{g}+\phi_{g}^{\tilde{p} l}\right)-\frac{1}{2} J_{g}^{\tilde{p} l}
\end{array}\right]
\end{aligned}
$$

Here,

$$
\begin{aligned}
& \alpha_{g}^{s+}=-\beta_{g} \kappa_{g} \cosh \left(\kappa_{g}\right)+\frac{1}{4} \sinh \left(\kappa_{g}\right) \text { and } \\
& \alpha_{g}^{c+}=-\beta_{g} \kappa_{g} \sinh \left(\kappa_{g}\right)+\frac{1}{4}\left(\cosh \left(\kappa_{g}\right)-\frac{\sinh \left(\kappa_{g}\right)}{\kappa_{g}}\right) .
\end{aligned}
$$

The resulting outgoing currents will be used as the incoming current of the neighboring node in the subsequent step.

\section{(2) Two-Node Kernel}

In the two-node configuration, the node average fluxes of the two nodes are specified as the constraints, and the simultaneous solution of all directions is not pursued. Therefore, the constant term in the particular solution given by Eq. (4) is valid and the even term coefficient of the homogeneous solution, $B_{g}$, is obtained from the node average flux constraint as

$$
B_{g}=\frac{\kappa_{g}}{\sinh \left(\kappa_{g}\right)}\left(\bar{\phi}_{g}-c_{g 0}\right) .
$$

Now, the only unknown coefficient is $A_{g}$ and it needs to be determined for each node leading to two unknowns per node. These two are determined by solving the following coupled equations that represent the flux and current continuity at the interface:

$$
\left[\begin{array}{cc}
\sinh \left(\kappa_{g}^{l}\right) & \sinh \left(\kappa_{g}^{r}\right) \\
2 \beta_{g}^{l} \kappa_{g}^{l} \cosh \left(\kappa_{g}^{l}\right) & -2 \beta_{g}^{r} \kappa_{g}^{r} \cosh \left(\kappa_{g}^{r}\right)
\end{array}\right]\left[\begin{array}{c}
A_{g}^{l} \\
A_{g}^{r}
\end{array}\right]=\left[\begin{array}{c}
\gamma_{\phi} \\
\gamma_{J}
\end{array}\right] .
$$

Here,

$$
\begin{aligned}
\gamma_{\phi}= & B_{g}^{r} \cosh \left(\kappa_{g}^{r}\right)-B_{g}^{l} \cosh \left(\kappa_{g}^{l}\right) \\
& +\sum_{i=0}^{4}\left((-1)^{i} c_{g i}^{r}-c_{g i}^{l}\right) \text { and } \\
\gamma_{J}= & -2 \beta_{g}^{l}\left(B_{g}^{l} \kappa_{g}^{l} \sinh \left(\kappa_{g}^{l}\right)+a_{g 1}^{l}+3 a_{g 2}^{l}+6 a_{g 3}^{l}+10 a_{g 4}^{l}\right) \\
& -2 \beta_{g}^{r}\left(B_{g}^{r} \kappa_{g}^{r} \sinh \left(\kappa_{g}^{r}\right)-a_{g 1}^{r}+3 a_{g 2}^{r}-6 a_{g 3}^{r}+10 a_{g 4}^{r}\right) .
\end{aligned}
$$

(3) Source Iteration Convergence Check

The source expansion nodal method involves a source iteration. It is thus necessary to establish a proper condition to check the convergence of the multigroup flux solution. Since the change in the flux shape is directly reflected into the fission source shape, monitoring the convergence of the fission source shape within the local nodes can be used as a means of checking the convergence. Instead of checking the fission source convergence, it is possible to check the convergence of individual group fluxes. But that kind of strict convergence check is not necessary because the source level and shape keep changing during the outer iteration. Specifically, the following convergence parameter is used in SENM:

$$
\delta^{(n+1)}=\frac{1}{\bar{\psi}^{(n+1)}}\left(\frac{1}{2} \int_{-1}^{1}\left(\psi^{(n+1)}(\xi)-\psi^{(n)}(\xi)\right)^{2} d \xi\right)^{1 / 2},
$$

with $n$ being the source iteration index.

By expanding the fission source into a quartic polynomial using Legendre polynomials, the above integral can be obtained easily as follows owing to the orthogonal property of the Legendre polynomials:

$$
\delta^{(n+1)}=\frac{1}{\bar{\psi}^{(n+1)}}\left(\sum_{i=0}^{4} \frac{1}{2 i+1}\left(q_{\psi i}^{(n+1)}-q_{\psi i}^{(n)}\right)^{2}\right)^{1 / 2}
$$

where $q_{\psi i}^{(n)}$ is the $i$-th-order fission source expansion coefficient at iteration step $n$. In the case of nonfuel nodes, the fission source is replaced with the lowest energy group flux.

\section{Flux Expansion with Quartic Polynomial and Expo- nential Functions}

The fourth-order source expansion results in the analytic flux solution given by Eq. (3). This flux solution suggests a flux expansion method that has the seven basis functions. In the flux expansion method, the same form of the flux distribution is used in the source term as well as the loss term, so that no source expansion is involved. This approach, 
however, requires a simultaneous solution of the expansion coefficients of all groups. The derivation to determine the group-coupled expansion coefficients in a two-node configuration was done by $\mathrm{Zimin}^{2)}$ and $\mathrm{Fu}^{6)}$ for the case employing a second-order polynomial and two exponential functions as the basis functions. The derivation below is to increase the order of the polynomial to four.

(1) Basis Functions and their Properties

As first introduced by Hennart, ${ }^{12)}$ it is convenient in the derivation to redefine the basis functions involving sinh and cosh such that they satisfy the condition of orthogonality with Legendre polynomials and the unity boundary value. Since the new basis functions would be group-dependent, it is necessary to generalize the basis functions such that all of them have group dependence. In this regard, let $P_{g i}(\xi) \equiv P_{i}(\xi)(0 \leq i \leq 4)$ and consider the following constraints:

$$
\begin{aligned}
& \int_{-1}^{1} P_{g i}(\xi) P_{g j}(\xi) d \xi=0,0 \leq i \leq 4,5 \leq j \leq 6 \\
& P_{g 5}( \pm 1)= \pm 1 \text { and } P_{g 6}( \pm 1)=1
\end{aligned}
$$

with the following two newly defined basis functions:

$$
\begin{aligned}
& P_{g 5}(\xi)=\frac{\sinh \left(\kappa_{g} \xi\right)+m_{g 1} P_{g 1}(\xi)+m_{g 3} P_{g 3}(\xi)}{\sinh \left(\kappa_{g}\right)+m_{g 1}+m_{g 3}} \text { and } \\
& P_{g 6}(\xi)=\frac{\cosh \left(\kappa_{g} \xi\right)+m_{g 0} P_{g 0}(\xi)+m_{g 2} P_{g 2}(\xi)+m_{g 4} P_{g 4}(\xi)}{\cosh \left(\kappa_{g}\right)+m_{g 0}+m_{g 2}+m_{g 4}}
\end{aligned}
$$

The requirements of Eq. (25) can all be satisfied by choosing the free parameter, $m_{g i}{ }^{\prime} s$ as follows:

$$
\begin{aligned}
k_{g, 20}= & 6, k_{g, 31}=10, k_{g, 40}=20, k_{g, 42}=14, \\
k_{g, 51}= & \frac{2\left(\kappa_{g} \cosh \left(\kappa_{g}\right)-\sinh \left(\kappa_{g}\right)+5 m_{g 3}\right)}{\sinh \left(\kappa_{g}\right)+m_{g 1}+m_{g 3}}, \\
k_{g, 53}= & \frac{2\left(\kappa_{g}\left(15+\kappa_{g}^{2}\right) \cosh \left(\kappa_{g}\right)-3\left(5+2 \kappa_{g}^{2}\right) \sinh \left(\kappa_{g}\right)\right)}{\kappa_{g}^{2}\left(\sinh \left(\kappa_{g}\right)+m_{g 1}+m_{g 3}\right)}, \\
k_{g, 60}= & \frac{2 \kappa_{g} \sinh \left(\kappa_{g}\right)+6 m_{g 2}+20 m_{g 4}}{\cosh \left(\kappa_{g}\right)+m_{g 0}+m_{g 2}+m_{g 4}}, \\
k_{g, 62}= & \frac{2\left(-3 \cosh \left(\kappa_{g}\right)+\left(3+\kappa_{g}^{2}\right) \frac{\sinh \left(\kappa_{g}\right)}{\kappa_{g}}+7 m_{g 4}\right)}{\cosh \left(\kappa_{g}\right)+m_{g 0}+m_{g 2}+m_{g 4}}, \\
k_{g, 64}= & 2\left(-5 \kappa_{g}\left(21+2 \kappa_{g}^{2}\right) \cosh \left(\kappa_{g}\right)+\left(105+45 \kappa_{g}^{2}+\kappa_{g}^{4}\right) \sinh \left(\kappa_{g}\right)\right) \\
\kappa_{g}^{3}\left(\cosh \left(\kappa_{g}\right)+m_{g 0}+m_{g 2}+m_{g 4}\right) & \text { and } \\
k_{g, i j}= & 0, \text { otherwise. }
\end{aligned}
$$

\section{(2) Balance Equation in Matrix Form}

Let the flux vector represent the group fluxes of all groups. Then, by using the matrix and vector notations, the flux expansion vector can be represented as

$$
\boldsymbol{\Phi}(\xi)=\overline{\boldsymbol{\Phi}}+\sum_{i=1}^{6} \mathbf{P}_{i}(\xi) \mathbf{c}_{i}
$$

where

$$
\begin{aligned}
m_{g 0}= & -\frac{\sinh \left(\kappa_{g}\right)}{\kappa_{g}}, \\
m_{g 1}= & \frac{3}{\kappa_{g}^{2}}\left(\sinh \left(\kappa_{g}\right)-\kappa_{g} \cosh \left(\kappa_{g}\right)\right), \\
m_{g 2}= & -\frac{5}{\kappa_{g}^{3}}\left(3 \sinh \left(\kappa_{g}\right)-3 \kappa_{g} \cosh \left(\kappa_{g}\right)+\kappa_{g}^{2} \sinh \left(\kappa_{g}\right)\right), \\
m_{g 3}= & \frac{7}{\kappa_{g}^{4}}\left(15 \sinh \left(\kappa_{g}\right)-15 \kappa_{g} \cosh \left(\kappa_{g}\right)\right. \\
& \left.+6 \kappa_{g}^{2} \sinh \left(\kappa_{g}\right)-\kappa_{g}^{3} \cosh \left(\kappa_{g}\right)\right), \text { and } \\
m_{g 4}= & -\frac{9}{\kappa_{g}^{5}}\left(105 \sinh \left(\kappa_{g}\right)-105 \kappa_{g} \cosh \left(\kappa_{g}\right)\right. \\
& \left.+45 \kappa_{g}^{2} \sinh \left(\kappa_{g}\right)-10 \kappa_{g}^{3} \cosh \left(\kappa_{g}\right)+\kappa_{g}^{4} \sinh \left(\kappa_{g}\right)\right) .
\end{aligned}
$$

For the weighted residual method (WRM) calculation to follow, the inner products of the basis functions and the secondorder derivatives are obtained as follows:

$$
\begin{aligned}
& \int_{-1}^{1} P_{g i}(\xi) P_{g j}(\xi) d \xi \\
& \quad=\mu_{i j}=\left\{\begin{array}{cc}
\frac{2}{2 i+1}, & i=j, 0 \leq i \leq 4 \\
0, & i \neq j, 0 \leq i \leq 6,0 \leq j \leq 4
\end{array}\right.
\end{aligned}
$$

and

$$
\int_{-1}^{1} P_{g i}{ }^{\prime \prime}(\xi) P_{g j}(\xi) d \xi=k_{g, i j}
$$

where

$$
\begin{aligned}
& \overline{\boldsymbol{\Phi}}=\left(\bar{\phi}_{1}, \bar{\phi}_{2}, \cdots, \bar{\phi}_{G}\right)^{T}, \\
& \boldsymbol{\Phi}(\xi)=\left(\phi_{1}(\xi), \phi_{2}(\xi), \cdots, \phi_{G}(\xi)\right)^{T}, \\
& \mathbf{P}_{i}(\xi)=\operatorname{diag}\left(P_{1 i}(\xi), P_{2 i}(\xi), \cdots, P_{G i}(\xi)\right), \text { and } \\
& \mathbf{c}_{i}=\left(c_{1 i}, c_{2 i}, \cdots, c_{G i}\right)^{T} .
\end{aligned}
$$

with $\mathrm{G}$ being the number of groups.

In terms of matrices and vectors, the nodal balance equation for all groups is represented as 


$$
-\mathbf{D} \Phi^{\prime \prime}(\xi)+\mathbf{A} \Phi(\xi)=-\mathbf{L}(\xi)
$$

where

$$
\begin{aligned}
& \mathbf{L}(\xi)=\left(L_{1}(\xi), L_{2}(\xi), \cdots, L_{G}(\xi)\right)^{T}, \\
& \mathbf{D}=\frac{4}{h^{2}} \operatorname{diag}\left(D_{1}, D_{2}, \cdots, D_{G}\right), \\
& \mathbf{\Sigma}_{r}=\operatorname{diag}\left(\Sigma_{r 1}, \Sigma_{r 2}, \cdots, \Sigma_{r G}\right)^{T}, \\
& \mathbf{F}=\left[\begin{array}{ccc}
\chi_{1} \nu \Sigma_{f, 1} & \cdots & \chi_{1} \nu \Sigma_{f, G} \\
\vdots & \ddots & \vdots \\
\chi_{G} v \Sigma_{f, 1} & \cdots & \chi_{G} v \Sigma_{f, G}
\end{array}\right], \\
& \boldsymbol{\Sigma}_{s}=\left[\begin{array}{cccc}
0 & \Sigma_{21} & \cdots & \Sigma_{G 1} \\
\Sigma_{12} & 0 & & \Sigma_{G 2} \\
\vdots & & \ddots & \vdots \\
\Sigma_{1 G} & \cdots & \Sigma_{G-1 G} & 0
\end{array}\right], \text { and }
\end{aligned}
$$

$\mathbf{A}=\boldsymbol{\Sigma}_{r}-\boldsymbol{\Sigma}_{s}-\lambda \mathbf{F}$.

Since the node average fluxes are all given, there are 6 coefficients in Eq. (30) to be determined per group and per node. These 6 coefficients can be determined by applying the weighted residual method (WRM) up to the fourth order and then by using the two interface conditions. In the following, the even term coefficients are determined first, which are not dependent on the other node.

(3) Even Term Coefficients

By applying the WRM with $P_{0}, P_{2}$, and $P_{4}$ to Eq. (31), namely,

$$
\begin{gathered}
-\mathbf{D} \int_{-1}^{1} \boldsymbol{\Phi}^{\prime \prime}(\xi) P_{i}(\xi) d \xi+\mathbf{A} \int_{-1}^{1} \boldsymbol{\Phi}(\xi) P_{i}(\xi) d \xi \\
=-\int_{-1}^{1} \mathbf{L}(\xi) P_{i}(\xi) d \xi, \quad i=0,2,4,
\end{gathered}
$$

the following relations are obtained:

$$
\begin{aligned}
& -\mathbf{D}\left(\mathbf{K}_{20} \mathbf{c}_{2}+\mathbf{K}_{40} \mathbf{c}_{4}+\mathbf{K}_{60} \mathbf{c}_{6}\right)+2 \mathbf{A} \overline{\boldsymbol{\Phi}}=-2 \mathbf{l}_{0}, \\
& -\mathbf{D}\left(\mathbf{K}_{42} \mathbf{c}_{4}+\mathbf{K}_{62} \mathbf{c}_{6}\right)+\mu_{22} \mathbf{A} \mathbf{c}_{2}=-\mu_{22} \mathbf{l}_{2}, \text { and } \\
& -\mathbf{D} \mathbf{K}_{64} \mathbf{c}_{6}+\mu_{44} \mathbf{A} \mathbf{c}_{4}=\mathbf{0},
\end{aligned}
$$

where $\mathbf{K}_{i j}=\operatorname{diag}\left(k_{1, i j}, k_{2, i j}, \cdots, k_{G, i j}\right)$. Note that only $\mathbf{A}$ is a $\mathrm{G} \times \mathrm{G}$ full matrix and all the other matrices are diagonal ones. Without using the inverse of $\mathbf{A}$, which would require a nontrivial amount of operations, Eq. (33) can be sequentially solved for the even coefficients as

$$
\begin{aligned}
\mathbf{c}_{6}= & \mu_{44} \mathbf{K}_{64}^{-1} \mathbf{D}^{-1} \mathbf{A} c_{4}, \\
\mathbf{c}_{2}= & \mathbf{K}_{20}^{-1}\left(2 \mathbf{D}^{-1}\left(\mathbf{l}_{0}+\mathbf{A} \overline{\boldsymbol{\Phi}}\right)-\mathbf{K}_{40} \mathbf{c}_{4}\right. \\
& \left.-\mu_{44} \mathbf{K}_{60} \mathbf{K}_{64}^{-1} \mathbf{D}^{-1} \mathbf{A} \mathbf{c}_{4}\right),
\end{aligned}
$$

and

$$
\left[\begin{array}{cccc}
\mathbf{I} & \mathbf{I}+\mathbf{H}_{l} & \mathbf{I} & \mathbf{I}+\mathbf{H}_{r} \\
\mu_{11} \mathbf{A}_{l} & -\mathbf{D}_{l}\left(\mathbf{K}_{31}^{l}+\mathbf{K}_{51}^{l} \mathbf{H}_{l}\right) & \mathbf{0} & \mathbf{0} \\
-\tilde{\mathbf{D}}_{l} & -6 \tilde{\mathbf{D}}_{l}-\tilde{\mathbf{D}}_{l} \mathbf{T}_{5}^{l} \mathbf{H}_{l} & \tilde{\mathbf{D}}_{r} & 6 \tilde{\mathbf{D}}_{r}+\tilde{\mathbf{D}}_{r} \mathbf{T}_{5}^{r} \mathbf{H}_{r} \\
\mathbf{0} & \mathbf{0} & \mu_{11} \mathbf{A}_{r} & -\mathbf{D}_{r}\left(\mathbf{K}_{31}^{r}+\mathbf{K}_{51}^{r} \mathbf{H}_{r}\right)
\end{array}\right]\left[\begin{array}{c}
\mathbf{c}_{1}^{l} \\
\mathbf{c}_{3}^{l} \\
\mathbf{c}_{1}^{r} \\
\mathbf{c}_{3}^{r}
\end{array}\right]=\left[\begin{array}{c}
\mathbf{b}_{\phi} \\
-\mu_{11} \mathbf{l}_{1}^{l} \\
\mathbf{b}_{J} \\
-\mu_{11} \mathbf{l}_{1}^{r}
\end{array}\right]
$$


where the subscripts $r$ and $l$ designate the right and left nodes of the interface and

$$
\begin{aligned}
\mathbf{b}_{\phi} & =\sum_{m=l, r}\left(\sum_{i=2,4,6} \mathbf{c}_{i}^{m}+\overline{\boldsymbol{\Phi}}_{m}\right), \\
\mathbf{b}_{J} & =\sum_{m=l, r} \tilde{\mathbf{D}}_{m}\left(3 \mathbf{c}_{2}^{m}+10 \mathbf{c}_{4}^{m}+\mathbf{T}_{6}^{m} \mathbf{c}_{6}^{m}\right), \text { and } \\
\mathbf{H} & =\mu_{33} \mathbf{K}_{53}^{-1} \mathbf{D}^{-1} \mathbf{A} .
\end{aligned}
$$

$\mathrm{Fu}$ and $\mathrm{Cho}^{6)}$ used the interface current vector as the final unknown vector and eliminated all the expansion coefficient vectors using the correlations between the current, flux and expansion coefficients so that, finally, a $\mathrm{G} \times \mathrm{G}$ linear system is realized. But this approach is not pursued here because this reduction involves numerous inverses of $\mathrm{G} \times \mathrm{G}$ matrices. Instead, we solve Eq. (42) directly by exploiting the sparse structure of the linear system.

\section{Two-Level Coarse Mesh Finite Formulation}

There can be two views of the coarse mesh finite difference (CMFD) formulation that employs a correction term in the current vs. node average flux relation. The first view is to regard the CMFD as an acceleration scheme for the higher order methods, whereas the other is to view it as a complete nodal balance formulation employing higher order current relations. The latter view enables more extensive and efficient applications of the CMFD formulation to various problems. For instance, a CMFD standalone spatial kinetics calculation is possible by applying a suitable temporal differencing to the CMFD balance equations. Only occasional updates of the higher order correction factors are required in this case. This view also enables a two-group representation of a multigroup problem with dynamically collapsed group constants as explained in the next section. The work here is based on the latter view.

The correction factor appearing in the current vs. node average flux relation can be obtained by solving the local problem in either one-node or two-node configuration. It can also be obtained by analytically solving for the current in terms of the two node average fluxes. ${ }^{13,14)}$ If the analytic nodal method is used in the two-node nodal kernel, then the same expression as the analytic CMFD (ACMFD) can be obtained.

With the one-node formulation, the partial currents are given as the boundary conditions so the node average group fluxes can be newly determined. With the one-node SENM kernel, there is an important advantage to avoid the $\mathrm{MG}$ CMFD formulation as will be explained in the following subsection. On the other hand, the MG CMFD calculation that is inevitable in the two-node formulation can be accelerated by a $2 \mathrm{G}$ CMFD as described in the second subsection.

\section{Two-Group CMFD Formulation with Multigroup One-Node Kernel}

Suppose that MG incoming partial currents are specified for a one-node problem. It can be solved for the MG node average fluxes and outgoing partial currents. It is then possible to define the following $2 \mathrm{G}$ cross section and the current correction factor:

$$
\Sigma_{\alpha \hat{g}}=\sum_{g \in S_{\hat{g}}} \Sigma_{\alpha g} \varphi_{g}
$$

and

$$
\hat{D}_{\hat{g}}=-\frac{\tilde{D}_{\hat{g}}\left(\bar{\phi}_{\hat{g}}^{r}-\bar{\phi}_{\hat{g}}^{l}\right)+j_{\hat{g}}}{\bar{\phi}_{\hat{g}}^{r}+\bar{\phi}_{\hat{g}}^{l}}
$$

where $\hat{g}$ is the coarse group index, $\alpha$ is the reaction type, and

$$
\varphi_{g}=\frac{\bar{\phi}_{g}}{\bar{\phi}_{\hat{g}}}, \bar{\phi}_{\hat{g}}=\sum_{g \in S_{\hat{g}}} \bar{\phi}_{g}, j_{\hat{g}}=\sum_{g \in S_{\hat{g}}} j_{g} \quad(\hat{g}=1,2) .
$$

Here, $S_{\hat{g}}$ designates the set of the fine group numbers belonging to coarse group $\hat{g}$. The coarse group current correction will be used in the $2 \mathrm{G}$ CMFD formulation to relate the interface current with the two node average fluxes of the interface as

$$
J_{\hat{g}}=-\tilde{D}_{\hat{g}}\left(\bar{\phi}_{\hat{g}}^{r}-\bar{\phi}_{\hat{g}}^{l}\right)-\hat{D}_{\hat{g}}\left(\bar{\phi}_{\hat{g}}^{r}+\bar{\phi}_{\hat{g}}^{l}\right),
$$

where $\tilde{D}_{\hat{g}}$ is the finite difference method (FDM)-based proportionality constant relating the current with the flux difference.

Once the $2 \mathrm{G}$ CMFD calculation is performed, the $2 \mathrm{G}$ node average flux distribution becomes available. In order to perform one-node MG nodal calculation based on the new $2 \mathrm{G}$ CMFD solution, the MG partial currents need to be properly determined from the $2 \mathrm{G}$ node average fluxes. Since the surface flux is necessary to determine the partial current, the surface flux must be determined from the two node average fluxes. This can be done by using a relation similarly formulated for the surface flux:

$$
\phi_{\hat{g}}^{s}=\omega_{\hat{g}} \bar{\phi}_{\hat{g}}^{l}+\left(1-\omega_{\hat{g}}\right) \bar{\phi}_{\hat{g}}^{r}+\hat{\omega}_{\hat{g}}\left(\bar{\phi}_{\hat{g}}^{r}+\bar{\phi}_{\hat{g}}^{l}\right),
$$

where $\omega_{\hat{g}}$ is the FDM-based weighting factor for the left node and $\hat{\omega}_{\hat{g}}$ is the correction factor for the surface flux that can be determined from the previous one-nodal solution employing Eq. (46) for the surface flux.

The $2 \mathrm{G}$ outgoing current obtained by using the net current and surface flux given by Eqs. (45) and (46) should now be expanded to multigroup values for use in the MG one-node calculation. This expansion can be done by using the outgoing current spectra determined in the previous nodal calculation as

$$
j_{g}^{\text {out }}=\varsigma_{g} j_{s \hat{g}}^{\text {out }}
$$

where the outgoing partial current spectrum is precalculated using the nodal results as

$$
\varsigma_{g}=\frac{j_{g}^{\text {out }}}{\sum_{g^{\prime} \in S_{\hat{g}}} j_{g^{\prime}}^{\text {out }}} .
$$

\section{Two-Level CMFD Formulation with Two-Node Ker- nels}

In the MG CMFD calculation with a two-node kernel, it is necessary to update only the current correction factor from the two-node nodal calculation. Then, the CMFD calculation to provide the updated node average fluxes can be performed in the multigroup structure only. However, the convergence 


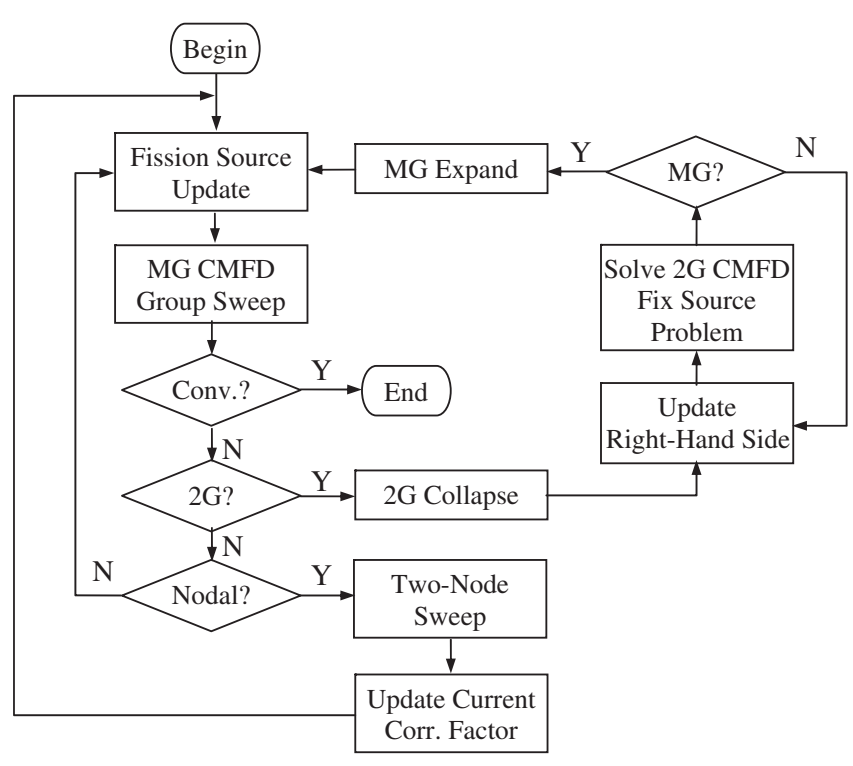

Fig. 1 Two-level CMFD calculation flow

of a multigroup problem is generally slower than the corresponding two-group problem because of the ineffectiveness of the source iteration scheme. For instance, the Chebyshev acceleration scheme employed in the MG eigenvalue problem performs much worse than the Wielandt eigenvalue shift method, which can be readily employed in a $2 \mathrm{G}$ problem. This motivates a dynamic $2 \mathrm{G}$ formulation during the $\mathrm{MG}$ CMFD calculation. Once the MG node average fluxes and interface currents become available, an equivalent $2 \mathrm{G}$ problem can be formulated easily by using Eqs. (43) and (44). Then, the CMFD calculation can be performed with the $2 \mathrm{G}$ formulation to produce a new $2 \mathrm{G}$ flux distribution, which is to be used to adjust the spatial shape of the MG flux. The expansion from the $2 \mathrm{G}$ node average fluxes to $\mathrm{MG}$ can be done using the intranode spectrum determined in the previous MG CMFD calculation. The subsequent MG CMFD is to adjust the MG nodal balance, and the updated MG fluxes are then fed into the MG two-node calculation for the update of the current correction factor. The two-level CMFD calculation flow involving $2 \mathrm{G}$ and MG CMFD solutions is depicted in Fig. 1.

As seen in the flow chart, there are several decision points in the two-level CMFD calculation. First, the overall convergence check is made only once per nodal update cycle. It is done at the first MG CMFD group sweep performed right after each nodal update. This is to make sure that the overall convergence is achieved even after the nodal update. The exit from the MG CMFD to 2G CMFD is made when sufficient residual error reduction is achieved. The exit from the $2 \mathrm{G}$ CMFD to MG CMFD is also made after a certain residual reduction during the $2 \mathrm{G}$ calculation. The exit from the $\mathrm{MG}$ to the nodal update is made at the first MG CMFD after a $2 \mathrm{G}$ CMFD calculation.

\section{Performance Examination}

In order to examine the performance of the multigroup solution methods presented above, a FORTRAN90 code
Table 1 Multigroup eigenvalue test problems

\begin{tabular}{cccccc}
\hline $\begin{array}{c}\text { Core } \\
\text { Type }\end{array}$ & Name & Gr & $\begin{array}{c}\text { Core } \\
\text { Lattice } \\
(\text { Full Core })\end{array}$ & $\begin{array}{c}\text { Assembly } \\
\text { Pitch } \\
(\mathrm{cm})\end{array}$ & $\begin{array}{c}\text { Core } \\
\text { Height } \\
(\mathrm{cm})\end{array}$ \\
\hline \multirow{1}{*}{ LWR } & IAEA3D & 2 & $17 \times 17 \times 19$ & 20.0 & 380 \\
\cline { 2 - 6 } & L336C5 & 2 & $6 \times 6 \times 1$ & 21.42 & - \\
\cline { 2 - 6 } & MOXTR2G & 2 & $17 \times 17 \times 22$ & 21.42 & 409 \\
\cline { 2 - 6 } & KOEST4G & 4 & $8 \times 8 \times 1$ & 21.0 & - \\
\cline { 2 - 6 } & MOXTR4G & 4 & $17 \times 17 \times 22$ & 21.42 & 409 \\
\cline { 2 - 6 } & L336C5G7 & 7 & $6 \times 6 \times 1$ & 21.42 & - \\
\cline { 2 - 6 } FBR & MOXTR8G & 8 & $17 \times 17 \times 22$ & 21.42 & 409 \\
\hline & SNR300 & 4 & $38 \times 38 \times 10$ & 5.4 & 175 \\
\cline { 2 - 6 } & TAKEDA3 & 4 & $64 \times 64 \times 14$ & 5.0 & 180 \\
\hline & PEACER & 25 & $28 \times 28 \times 12$ & 20.4 & 255 \\
\hline
\end{tabular}

named RENUS (Reactor Numerical Simulator) was written and it was applied to a wide range of multigroup eigenvalue benchmark problems that span from various LWR cores involving severe thermal flux gradients and large FBR cores with a very heterogeneous configuration. Since the performance of a solver should be evaluated in terms of not only speed but also accuracy, the solution error of the three SANM nodal kernels is assessed first by examining the node size dependence of the error. For this assessment, a reference solution for each problem was generated by employing a very fine node structure (64 nodes/assembly). Additional examination of the solution accuracy is made to evaluate the impact of the 4th-order source expansion as opposed to the 2nd order. The execution performance in terms of the number of nodal updates, CMFD iterations, and computing time is then examined. Finally, the acceleration performance of the 2G CMFD formulation is assessed by comparing with the MG only cases.

The test problems used here consist of well-known benchmark problems except the last one. The number of energy groups ranges from 2 to 24 and the flux spectrum represents either an LWR or an FBR. Table 1 lists the characteristics of the test problems. Cross section data and geometry are all given in the cited papers for IAEA3D, ${ }^{15)}$ MOXTR2 $\mathrm{G}^{16)}$ through MOXTR8G, KAIST4G, ${ }^{17)}$ KOEBERG, ${ }^{18)}$ SNR300, ${ }^{19)}$ and TAKEDA3 ${ }^{20)}$ problems. There are three cases of the MOX core transient benchmark problem ${ }^{16)}$ with different numbers of groups. These cases represent the same core condition, which is a hot-zero-power (HZP) rodded condition designated by Part 3 of the benchmark. The boron concentration is set to $1334 \mathrm{pcm}$, which is the critical boron concentration found in the PARCS 8G case as indicated in the benchmark report. For L336C $5^{21)}$ and L336C5G7 ${ }^{22)}$ which are originally pin-cell-level heterogeneous problems, the assembly-homogenized cross sections were not available in the benchmark specification. Therefore, the homogenized cross sections were made through 
assembly transport calculations. The TAKEDA3 problem represents one of the transport benchmark problems proposed by Takeda. Specifically, this case represents the rodded case of the axially heterogeneous large fast breeder reactor problem (Model 3). The PEACER ${ }^{23}$ ) problem is for a transmutation reactor core that has a pancake shape. The 24-group cross sections of this core are not yet publicly available. This problem is included for the sake of demonstrating the execution performance.

\section{Solution Accuracy}

In order to examine the solution accuracy and its dependence on the node size, the eigenvalue calculations were performed with all three kernels and with various node sizes. The errors in the eigenvalue and assembly power distribution of these cases are given in Table 2 . The case identifiers $1 \mathrm{~N}$ SENM and 2N-SENM in the table represent the one-node and two-node kernels with source expansion, respectively, which involves source iterations, while 2N-SANM is for the two-node kernel with flux expansion, which involves the direct solution of $4 \mathrm{G} \times 4 \mathrm{G}$ linear systems.

It is first noted in Table 2 that all the three kernels have essentially the same accuracy. In fact, there should be no difference between $1 \mathrm{~N}$ and $2 \mathrm{~N}$ SENM because they use the same form of the source and flux solution. The $2 \mathrm{~N}$ SANM is, however, expected to be more accurate than the $2 \mathrm{~N}-\mathrm{SENM}$ because it has the exponential terms in the source expansion as well. But the negligible difference implies that the 4th-order polynomial expansion of the source without the exponential terms is accurate enough.

In all the cases, the eigenvalue error of the $1 \times 1$ SANM cases is less than $50 \mathrm{pcm}$ even for the L336C5 case, which involves a severe flux distortion due to the mixed loading of uranium and MOX fuel in a small $4 \times 4$ core. With 4 nodes per assembly, there is essentially no error with the SANM since the eigenvalue and the assembly power error are less than $10 \mathrm{pcm}$ and $0.4 \%$, respectively. This demonstrates the superior accuracy of the SANM kernels even for highly heterogeneous cores having locally strong absorbers.

In order to examine the effect of the higher order expansion of the source, the 2nd-order source expansion cases were performed with two-node kernels. The results are compared with the corresponding 4th-order expansion cases in Table 3. As shown in the table, the 2nd-order expansion is sufficient in most cases except for the L336C5 cases. Particularly, for the L336C5G7 case, the consequence of using a lower order appears to be large for the 1 node/assembly case. This is because the thermal flux gradients appearing in the MOX fuel assembly become more severe with a finer group structure and it cannot be caught accurately enough with the 2nd-order expansion of the source. Since the computational work being increased due to the increase in the source expansion order by two is negligible, it would be better to retain the fourth order for generic applicability without sacrificing accuracy.

\section{Two-Level CMFD Calculation Performance}

The two-level CMFD calculation involves MG CMFD,
2G CMFD, and nodal kernel calculations. The three major calculation modules are coordinated by the calculation control scheme depicted in Fig. 1. The number of nodal kernel calculations can be significantly reduced by the two-level CMFD calculations. This is demonstrated using Table 4, which shows the number of iterations and computation time of each part. The results shown in this table were obtained from quarter core cases with 4 nodes/assembly. The residual reduction criteria to exit the MG CMFD and 2G CMFD were set to 0.5 and 0.1 , respectively. The overall convergence criterion was $10^{-5}$ for the global fission source error and the source shape convergence criterion needed in the SENM nodal kernels for the convergence check of Eq. (24) was 0.01. For the solution of the CMFD problems, the BiCGSTAB Krylov subspace method employing the blockwise incomplete LU preconditioning ${ }^{24)}$ was used in RENUS. The computing times were obtained as the average of 10 executions on a LINUX machine equipped with a $3.0 \mathrm{GHz}$ Intel Xeon CPU.

In Table 4, the first thing to note is that the average number of group sweeps per node is about double the number of nodal updates in the case of 2N-SENM. This means that only about two source updates are necessary per two-node problem. For $1 \mathrm{~N}-\mathrm{SENM}$, however, the average number of group sweeps is more than 4 times the nodal updates. This is because the spatial sweeps to update the incoming currents are additionally required in the case of one-node formulation to achieve a similar extent of error reduction. The need for the additional spatial sweeps is the reason for the more nodal computation time needed for the $1 \mathrm{~N}-\mathrm{SENM}$ than for the $2 \mathrm{~N}$ SENM. Compared with 2N-SENM, 2N-SANM performs even better at least for the $2 \mathrm{G}$ problems. This is because it is possible to obtain the inverse of a $2 \times 2$ matrix quite easily for 2 groups so that direct SANM can be performed better than the iterative SANM or the SENM. However, for more than 4 groups, the SENM outperforms the direct SANM and its effectiveness increases as the number of groups increases.

The fundamental reason behind this superior performance of the SENM is that only gradual improvement of the source shape is sufficient during the converging process. By using the previous flux shape obtained in the previous nodal update, it is possible to construct a quite good source shape that can be improved by a few additional source iterations at the current step. On the other hand, the source shape is determined very accurately in the direct SANM for the given transverse leakage distribution and eigenvalue by resolving completely all the group coupling effects. The complete determination of the accurate source shape might be a waste during the course of the converging process because the transverse leakage and eigenvalue are yet to be converged. This resembles the compensation between the outer and inner iterations in the standard nested eigenvalue calculation in which only partial convergence of the inner iteration is sufficient.

The effect of the two-level CMFD acceleration employing the additional $2 \mathrm{G}$ formulation is demonstrated in Table $\mathbf{5}$ for selected problems. If the $2 \mathrm{G}$ acceleration is off, the $\mathrm{MG}$ CMFD is to be accelerated only by the Chebyshev scheme. 
Table 2 Eigenvalue error $(\Delta \rho)$ and assembly power error variation with node size

\begin{tabular}{|c|c|c|c|c|c|c|c|c|}
\hline \multirow[b]{2}{*}{ Problem } & \multirow[b]{2}{*}{ Kernel } & \multicolumn{2}{|c|}{$1 \times 1$} & \multicolumn{2}{|c|}{$2 \times 2$} & \multicolumn{2}{|c|}{$4 \times 4$} & \multirow{2}{*}{$\begin{array}{c}\text { Reference } \\
\text { k-eff }\end{array}$} \\
\hline & & $\begin{array}{l}\text { k-eff } \\
(\mathrm{pcm})\end{array}$ & $\begin{array}{c}\text { power } \\
(\%)\end{array}$ & $\begin{array}{l}\text { k-eff } \\
(\mathrm{pcm})\end{array}$ & $\begin{array}{c}\text { power } \\
(\%)\end{array}$ & $\begin{array}{l}\text { k-eff } \\
(\mathrm{pcm})\end{array}$ & $\begin{array}{c}\text { power } \\
(\%)\end{array}$ & \\
\hline \multirow{3}{*}{ IAEA3D } & 1N-SENM & 10 & 1.16 & 2 & 0.41 & 0 & 0.05 & \multirow{3}{*}{1.02907} \\
\hline & 2N-SENM & 10 & 1.22 & 2 & 0.41 & 0 & 0.05 & \\
\hline & 2N-SANM & 10 & 1.16 & 2 & 0.35 & 0 & 0.05 & \\
\hline \multirow{3}{*}{ L336C5 } & 1N-SENM & 26 & 0.09 & 8 & 0.08 & 2 & 0.03 & \multirow{3}{*}{0.93882} \\
\hline & 2N-SENM & 26 & 0.10 & 8 & 0.08 & 2 & 0.03 & \\
\hline & 2N-SANM & 26 & 0.13 & 8 & 0.08 & 2 & 0.03 & \\
\hline \multirow{3}{*}{ MOXTR2G } & 1N-SENM & 35 & 1.16 & 6 & 0.18 & 1 & 0.04 & \multirow{3}{*}{1.00035} \\
\hline & 2N-SENM & 35 & 1.15 & 6 & 0.18 & 1 & 0.04 & \\
\hline & $2 \mathrm{~N}-\mathrm{SANM}$ & 35 & 1.15 & 6 & 0.18 & 1 & 0.04 & \\
\hline \multirow{3}{*}{ KAIST4G } & 1N-SENM & 24 & 0.28 & 8 & 0.10 & 1 & 0.03 & \multirow{3}{*}{1.06727} \\
\hline & 2N-SENM & 25 & 0.28 & 8 & 0.10 & 1 & 0.03 & \\
\hline & 2N-SANM & 25 & 0.28 & 8 & 0.10 & 1 & 0.03 & \\
\hline \multirow{3}{*}{ KOEBERG } & 1N-SENM & 42 & 2.28 & 4 & 0.23 & 1 & 0.11 & \multirow{3}{*}{1.00796} \\
\hline & 2N-SENM & 42 & 2.32 & 4 & 0.26 & 1 & 0.06 & \\
\hline & 2N-SANM & 42 & 2.32 & 4 & 0.26 & 1 & 0.06 & \\
\hline \multirow{3}{*}{ MOXTR4G } & $1 \mathrm{~N}-\mathrm{SENM}$ & 36 & 1.18 & 6 & 0.18 & 1 & 0.09 & \multirow{3}{*}{1.00017} \\
\hline & 2N-SENM & 36 & 1.18 & 6 & 0.18 & 1 & 0.09 & \\
\hline & 2N-SANM & 36 & 1.18 & 6 & 0.18 & 1 & 0.09 & \\
\hline \multirow{3}{*}{ L336C5G7 } & $1 \mathrm{~N}-\mathrm{SENM}$ & 46 & 0.85 & 2 & 0.05 & 0 & 0.02 & \multirow{3}{*}{1.18422} \\
\hline & 2N-SENM & 46 & 0.85 & 2 & 0.04 & 0 & 0.02 & \\
\hline & 2N-SANM & 46 & 0.85 & 2 & 0.04 & 0 & 0.02 & \\
\hline \multirow{3}{*}{ MOXTR8G } & 1N-SENM & 36 & 1.16 & 6 & 0.18 & 1 & 0.10 & \multirow{3}{*}{1.00004} \\
\hline & 2N-SENM & 36 & 1.18 & 6 & 0.18 & 1 & 0.09 & \\
\hline & 2N-SANM & 36 & 1.18 & 6 & 0.18 & 1 & 0.09 & \\
\hline \multirow{3}{*}{ SNR300 } & 1N-SENM & 4 & 0.17 & 0 & 0.08 & - & - & \multirow{3}{*}{1.01313} \\
\hline & 2N-SENM & 4 & 0.17 & 0 & 0.08 & 0 & 0.00 & \\
\hline & 2N-SANM & 4 & 0.17 & 0 & 0.08 & 0 & 0.06 & \\
\hline \multirow{3}{*}{ TAKEDA3 } & 1N-SENM & 10 & 0.52 & 3 & 0.12 & - & - & \multirow{3}{*}{0.96464} \\
\hline & 2N-SENM & 10 & 0.52 & 3 & 0.13 & 0 & 0.00 & \\
\hline & 2N-SANM & 10 & 0.52 & 3 & 0.13 & 0 & 0.00 & \\
\hline \multirow{3}{*}{ PEACER } & 1N-SENM & 26 & 0.35 & 3 & 0.05 & - & - & \\
\hline & 2N-SENM & 26 & 0.33 & 3 & 0.05 & 0 & 0.00 & 1.03777 \\
\hline & 2N-SANM & 26 & 0.33 & 3 & 0.05 & 0 & 0.00 & \\
\hline
\end{tabular}

But it cannot be effective in the CMFD calculation because the Chebyshev cycle should be reset after each nodal update, which leads to the deterioration of the Chebyshev acceleration performance. As shown in Table 5, the total computation time is reduced by more than half using the $2 \mathrm{G}$ CMFD acceleration for the MOXTR problems, which represents a large LWR having a large dominance ratio. But for the fast reactor problems and small LWRS having small dominance ratios, there is only a marginal improvement with the additional $2 \mathrm{G}$ CMFD or its performance is even worth in the case 
Table 3 Dependence of solution error on source expansion order

\begin{tabular}{|c|c|c|c|c|c|c|c|}
\hline \multirow[b]{2}{*}{ Problem } & \multirow{2}{*}{$\begin{array}{c}\text { Source } \\
\text { Exp. } \\
\text { Order }\end{array}$} & \multicolumn{2}{|c|}{$1 \times 1$} & \multicolumn{2}{|c|}{$2 \times 2$} & \multicolumn{2}{|c|}{$4 \times 4$} \\
\hline & & $\begin{array}{l}\text { k-eff } \\
(\mathrm{pcm})\end{array}$ & $\begin{array}{c}\text { power } \\
(\%)\end{array}$ & $\begin{array}{l}\text { k-eff } \\
(\mathrm{pcm})\end{array}$ & $\begin{array}{c}\text { power } \\
(\%)\end{array}$ & $\begin{array}{l}\text { k-eff } \\
(\mathrm{pcm})\end{array}$ & $\begin{array}{c}\text { power } \\
(\%)\end{array}$ \\
\hline \multirow{2}{*}{ IAEA3D } & 2 & 4 & 0.59 & 2 & 0.35 & 0 & 0.04 \\
\hline & 4 & 10 & 1.22 & 2 & 0.41 & 0 & 0.05 \\
\hline \multirow{2}{*}{ L336C5 } & 2 & -60 & 0.76 & -2 & 0.04 & 2 & 0.03 \\
\hline & 4 & 26 & 0.10 & 8 & 0.08 & 2 & 0.03 \\
\hline \multirow{2}{*}{ MOXTR2G } & 2 & 33 & 1.13 & 5 & 0.17 & 1 & 0.03 \\
\hline & 4 & 35 & 1.15 & 6 & 0.18 & 1 & 0.04 \\
\hline \multirow{2}{*}{ KAIST4G } & 2 & 21 & 0.29 & 9 & 0.08 & 1 & 0.03 \\
\hline & 4 & 25 & 0.28 & 8 & 0.10 & 1 & 0.03 \\
\hline \multirow{2}{*}{ KOEBERG } & 2 & 39 & 2.15 & 4 & 0.26 & 1 & 0.06 \\
\hline & 4 & 42 & 2.32 & 4 & 0.26 & 1 & 0.06 \\
\hline \multirow{2}{*}{ MOXTR4G } & 2 & 34 & 1.11 & 5 & 0.17 & 0 & 0.09 \\
\hline & 4 & 36 & 1.18 & 6 & 0.18 & 1 & 0.09 \\
\hline \multirow{2}{*}{ L336C5G7 } & 2 & 121 & 2.28 & 31 & 0.51 & 2 & 0.07 \\
\hline & 4 & 46 & 0.85 & 2 & 0.04 & 0 & 0.02 \\
\hline \multirow{2}{*}{ MOXTR8G } & 2 & 39 & 1.31 & 7 & 0.35 & 1 & 0.09 \\
\hline & 4 & 36 & 1.18 & 6 & 0.18 & 1 & 0.09 \\
\hline \multirow{2}{*}{ SNR300 } & 2 & 4 & 0.17 & 0 & 0.08 & 0 & 0.06 \\
\hline & 4 & 4 & 0.17 & 0 & 0.08 & 0 & 0.00 \\
\hline \multirow{2}{*}{ TAKEDA3 } & 2 & 10 & 0.52 & 3 & 0.13 & 0 & 0.00 \\
\hline & 4 & 10 & 0.52 & 3 & 0.13 & 0 & 0.00 \\
\hline \multirow{2}{*}{ PEACER } & 2 & 26 & 0.35 & 3 & 0.05 & 0 & 0.02 \\
\hline & 4 & 26 & 0.33 & 3 & 0.05 & 0 & 0.00 \\
\hline
\end{tabular}

of the two $4 \mathrm{G}$ fast reactor problems. This means that for cores with small dominance ratios, say less than 0.92 , the MG CMFD only is sufficient. The dominance ratios shown in the tables were obtained by performing additional power iterations after a converged solution is obtained.

\section{Conclusions}

In order to establish an efficient multigroup nodal calculation method, which would be fast, yet accurate, one- and two-node nodal kernels based on the fourth-order source expansion form of SANM were derived and implemented in a two-level CMFD formulation, which consists of multigroup and two-group CMFD modules. A direct SANM for resolving the group coupling completely at every nodal step was also derived by employing a quartic polynomial and exponential functions in the flux expansion.

In the source expansion formulation, source iterations are required during the nodal solution process, and thus, a proper fission source convergence checking scheme was established based on the orthogonality of the Legendre polynomials.
Through the various benchmark problems, it was demonstrated that only a few source iterations are enough for each two-node calculation regardless of the reactor type, fast or thermal. However, the need for the additional spatial sweeps in the one-node formulation increases the number of source iterations by more than double. This makes the one-node formulation inferior to the two-node formulation even though the one-node formulation has the advantage of simpler implementation and the possibility of the direct 2G CMFD formulation without an MG CMFD.

In the comparison of the direct vs. iterative formulations, it was shown that the direct SANM is better only for $2 \mathrm{G}$ problems because of the trivial work needed to calculate the inverse of $2 \times 2$ matrices. For MG problems, the iterative formulation or the source expansion formulation is much better than the direct one and its superiority increases significantly with the number of groups. The fundamental reason behind the superior performance of the iterative formulation is that only gradual improvement of the source shape is sufficient during the converging process.

The two-level CMFD turned out to be quite effective par- 
Table 4 Number of iterations and computing time

\begin{tabular}{|c|c|c|c|c|c|c|c|c|}
\hline \multirow{2}{*}{ Problem } & \multirow{2}{*}{ Kernel } & \multicolumn{2}{|c|}{ Nodal } & \multicolumn{2}{|c|}{ CMFD } & \multirow{2}{*}{$\begin{array}{c}\text { Total } \\
\text { Time } \\
(\mathrm{s})\end{array}$} & \multirow{2}{*}{$\begin{array}{c}\text { Nodal } \\
\text { Time } \\
(\mathrm{s})\end{array}$} & \multirow{2}{*}{$\begin{array}{c}\text { CMFD } \\
\text { Time } \\
\text { (s) }\end{array}$} \\
\hline & & Upd & $\mathrm{Swp}^{1)}$ & MG & $2 \mathrm{G}$ & & & \\
\hline \multirow{3}{*}{ IAEA3D } & $1 \mathrm{~N}-\mathrm{SENM}$ & 10 & 39 & 0 & 41 & 3.44 & 2.55 & 0.71 \\
\hline & 2N-SENM & 9 & 18 & 0 & 35 & 2.49 & 1.75 & 0.62 \\
\hline & 2N-SANM & 8 & 8 & 0 & 31 & 1.51 & 0.84 & 0.54 \\
\hline \multirow{3}{*}{ L336C5 } & 1N-SENM & 5 & 20 & 0 & 13 & 0.019 & 0.001 & 0.001 \\
\hline & 2N-SENM & 7 & 14 & 0 & 17 & 0.015 & 0.002 & 0.001 \\
\hline & 2N-SANM & 8 & 8 & 0 & 19 & 0.014 & 0.001 & 0.001 \\
\hline \multirow{3}{*}{ MOXTR2G } & 1N-SENM & 7 & 28 & 0 & 29 & 0.81 & 0.61 & 0.13 \\
\hline & 2N-SENM & 7 & 14 & 0 & 28 & 0.59 & 0.40 & 0.13 \\
\hline & 2N-SANM & 6 & 6 & 0 & 25 & 0.35 & 0.18 & 0.11 \\
\hline \multirow{3}{*}{ KAIST4G } & 1N-SENM & 6 & 31 & 23 & 15 & 0.020 & 0.007 & 0.004 \\
\hline & 2N-SENM & 7 & 14 & 27 & 18 & 0.019 & 0.005 & 0.004 \\
\hline & 2N-SANM & 7 & 7 & 27 & 18 & 0.024 & 0.010 & 0.004 \\
\hline \multirow{3}{*}{ KOEBERG } & 1N-SENM & 5 & 17 & 20 & 14 & 0.037 & 0.013 & 0.009 \\
\hline & 2N-SENM & 4 & 8 & 17 & 12 & 0.033 & 0.010 & 0.007 \\
\hline & 2N-SANM & 4 & 4 & 17 & 12 & 0.044 & 0.020 & 0.007 \\
\hline \multirow{3}{*}{ MOXTR4G } & 1N-SENM & 6 & 33 & 21 & 20 & 1.96 & 1.50 & 0.34 \\
\hline & 2N-SENM & 5 & 10 & 19 & 16 & 0.94 & 0.57 & 0.30 \\
\hline & 2N-SANM & 5 & 5 & 18 & 16 & 1.37 & 1.00 & 0.29 \\
\hline \multirow{3}{*}{ L336C5G7 } & 1N-SENM & 6 & 24 & 25 & 12 & 0.019 & 0.005 & 0.003 \\
\hline & 2N-SENM & 8 & 18 & 30 & 17 & 0.021 & 0.006 & 0.004 \\
\hline & 2N-SANM & 10 & 10 & 33 & 21 & 0.041 & 0.022 & 0.005 \\
\hline \multirow{3}{*}{ MOXTR8G } & $1 \mathrm{~N}-\mathrm{SENM}$ & 6 & 32 & 23 & 20 & 4.22 & 3.37 & 0.64 \\
\hline & 2N-SENM & 6 & 14 & 25 & 20 & 2.39 & 1.58 & 0.68 \\
\hline & 2N-SANM & 5 & 5 & 18 & 16 & 4.23 & 3.57 & 0.51 \\
\hline \multirow{3}{*}{ SNR300 } & 1N-SENM & 7 & 42 & 24 & 21 & 6.16 & 4.92 & 0.99 \\
\hline & 2N-SENM & 6 & 12 & 20 & 12 & 2.75 & 1.81 & 0.78 \\
\hline & 2N-SANM & 6 & 6 & 20 & 12 & 4.17 & 3.22 & 0.78 \\
\hline \multirow{3}{*}{ TAKEDAM3 } & 1N-SENM & 5 & 30 & 21 & 16 & 20.46 & 15.29 & 4.19 \\
\hline & 2N-SENM & 5 & 10 & 18 & 16 & 10.75 & 6.39 & 3.75 \\
\hline & 2N-SANM & 5 & 5 & 18 & 16 & 15.19 & 10.94 & 3.60 \\
\hline \multirow{3}{*}{ PEACER } & $1 \mathrm{~N}-\mathrm{SENM}$ & 5 & 26 & 17 & 11 & 15.43 & 11.92 & 2.00 \\
\hline & 2N-SENM & 6 & 12 & 20 & 18 & 10.85 & 7.36 & 2.39 \\
\hline & 2N-SANM & 6 & 6 & 20 & 18 & 61.14 & 57.33 & 2.39 \\
\hline
\end{tabular}

${ }^{1)}$ Average number of group sweeps per node

ticularly for the problems involving high dominance ratios such as in the case of large LWRs. From the observations stated above, it can be concluded that the two-level CMFD formulation with the two-node iterative SANM kernel provides a very efficient multigroup nodal calculation capability since it can generate solutions without wasting computational operations while retaining superior solution accuracy.

On the other hand, the one-node SENM concept, which is characterized by an MG nodal kernel and a direct $2 \mathrm{G}$ CMFD, can be used efficiently for other configurations in 
Table 5 Effectiveness of two-group CMFD acceleration

\begin{tabular}{|c|c|c|c|c|c|c|c|c|c|}
\hline \multirow{2}{*}{ Problem } & \multirow{2}{*}{ 2G CMFD } & \multicolumn{2}{|c|}{ Nodal } & \multicolumn{2}{|c|}{ CMFD } & \multirow{2}{*}{$\begin{array}{c}\text { Total } \\
\text { Time } \\
\text { (s) }\end{array}$} & \multirow{2}{*}{$\begin{array}{l}\text { Nodal } \\
\text { Time } \\
\text { (s) }\end{array}$} & \multirow{2}{*}{$\begin{array}{l}\text { CMFD } \\
\text { Time } \\
\text { (s) }\end{array}$} & \multirow{2}{*}{$\begin{array}{c}\text { Dominance } \\
\text { Ratio }\end{array}$} \\
\hline & & Upd. & Swp. & MG & $2 \mathrm{G}$ & & & & \\
\hline \multirow{2}{*}{ KAIST4G } & ON & 7 & 14 & 27 & 18 & 0.019 & 0.005 & 0.005 & \multirow{2}{*}{0.8256} \\
\hline & OFF & 7 & 14 & 40 & 0 & 0.019 & 0.005 & 0.004 & \\
\hline \multirow{2}{*}{ KOEBERG } & ON & 4 & 8 & 17 & 12 & 0.034 & 0.010 & 0.008 & \multirow{2}{*}{0.9595} \\
\hline & OFF & 5 & 10 & 60 & 0 & 0.046 & 0.013 & 0.017 & \\
\hline \multirow{2}{*}{ MOXTR4G } & ON & 5 & 10 & 19 & 16 & 0.95 & 0.57 & 0.30 & \multirow{2}{*}{0.9753} \\
\hline & OFF & 7 & 14 & 97 & 0 & 1.82 & 0.80 & 0.94 & \\
\hline \multirow{2}{*}{ MOXTR8G } & ON & 6 & 14 & 25 & 20 & 2.40 & 1.58 & 0.68 & \multirow{2}{*}{0.9849} \\
\hline & OFF & 9 & 21 & 132 & 0 & 5.10 & 2.31 & 2.65 & \\
\hline \multirow{2}{*}{ SNR300 } & ON & 6 & 12 & 20 & 12 & 2.75 & 1.81 & 0.78 & \multirow{2}{*}{0.7300} \\
\hline & OFF & 6 & 12 & 23 & 0 & 2.59 & 1.81 & 0.62 & \\
\hline \multirow{2}{*}{ TAKEDA3 } & ON & 5 & 10 & 18 & 16 & 10.69 & 6.39 & 3.70 & \multirow{2}{*}{0.9068} \\
\hline & OFF & 4 & 8 & 26 & 0 & 8.96 & 5.07 & 3.27 & \\
\hline \multirow{2}{*}{ PEACER } & ON & 6 & 12 & 20 & 18 & 10.80 & 7.34 & 2.39 & \multirow{2}{*}{0.9103} \\
\hline & OFF & 6 & 12 & 32 & 0 & 11.60 & 7.35 & 3.19 & \\
\hline
\end{tabular}

which the two-node formulation is cumbersome, such as the hexagonal problems. It is conceivable to use the two-dimensional source expansion in the one-node configuration in such cases. It is expected that the two-level CMFD formulation with a multigroup SENM kernel will provide a very efficient multigroup kinetics capability as well because it is possible to utilize a conditional nodal update scheme in the transient calculation by performing most of the transient calculations with CMFD only.

\section{Acknowledgement}

This work was financially supported by the Korean Ministry of Commerce, Industry and Energy through the IERC program funding NUTRECK.

\section{References}

1) P. D. Esser, K. S. Smith, "A semi-analytic two-group nodal model for SIMULATE-3," Trans. Am. Nucl. Soc., 68, 220 (1993).

2) V. G. Zimin, H. Ninokata, L. R. Pogosbekyan, "Polynomial and semi-analytic nodal methods for nonlinear iteration procedure," Proc. Int. Conf. Phys. Nucl. Sci. Tech. (PHSOR-98), Long Island, NY, October 5-8, 1998, 2, 994 (1998).

3) V. G. Zimin, H. Ninokata, "Nodal neutron kinetics model based on nonlinear iteration procedure for LWR analysis," Ann. Nucl. Energy, 25, 507 (1998).

4) C. Liao, Z. Xie, "The coupled kinetics and thermal-hydraulic three-dimensional code system NLSANMT/COBRA-IV for PWR core transient analysis," Ann. Nucl. Energy, 30, 405 (2003).
5) Y. I. Kim, Y. J. Kim, S. J. Kim, T. K. Kim, "A semi-analytic multigroup nodal method," Ann. Nucl. Energy, 26, 699 (1999).

6) X. D. Fu, N. Z. Cho, "Nonlinear analytic and semi-analytic nodal methods for multigroup neutron diffusion calculations," J. Nucl. Sci. Technol., 39, 1015 (2002).

7) T. Sutton, B. Aviles, "Diffusion theory methods for spatial kinetics calculations," Prog. Nucl. Energy, 30, 119-182, 141 (1996).

8) D. J. Lee, T. J. Downar, Y. H. Kim, "Convergence analysis of the nonlinear coarse-mesh finite difference method for onedimensional fixed-source neutron diffusion problem," Nucl. Sci. Eng., 147, 127 (2004).

9) H. G. Joo et al., "Multi-group transient calculation within the framework of a two-group hexagonal CMFD formulation," Proc. M\&C 2001, ANS Topical Mtg. on Math. Comp., Salt Lake City, U.S.A., Sept. 9-13, 2001, CDROM (2001).

10) T. Y. Han, H. G. Joo, C. H. Kim, "Two-group CMFD accelerated multi-group calculation with a semi-analytic nodal kernel," Proc. PHYSOR 2006, ANS Topical Mtg. on Reactor Physics, Vancouver, B.C., Canada, Sept. 10-14, 2006, CDROM (2006).

11) H. C. Lee, H. K. Joo, C. H. Kim, "A comparison between unified nodal method and semi-analytic nodal method for twonode multi-group neutron diffusion problem," Proc. Korean Nuclear Society 2004 Fall Mtg, Yongpyeong, Korea, Oct. 28-29, 2004, CDROM (2004), [in Korean].

12) J. P. Hennart, "On numerical analysis of analytical nodal methods," Num. Meth. Part. Diff. Eqn., 4, 233 (1988).

13) Y. A. Chao, "A theoretical analysis of the coarse mesh finite difference representation in advanced nodal methods," Proc. Math. and Comp., Reac. Phys. Environ. Anal. Nucl. Appl. (M\&C99), Madrid, Spain, Sept. 27-30, 1999, CDROM (1999).

14) J. M. Aragones, Carol Ahnert, Nuria Garcia-Herranz, "The an- 
alytic coarse-mesh finite difference method for multigroup and multidimensional diffusion calculations," Nucl. Sci. Eng., 157, 1 (2007).

15) "Benchmark Problem Book," ANL-7416, Supplement 2, Argonne National University (1977).

16) T. Kozlowski, T. Downar, "Pressurized water reactor MOX/ $\mathrm{UO}_{2}$ core transient benchmark, final report," NEA/NSC/ DOC(2006)20, OECD Nuclear Energy Agency (2006).

17) N. Z. Cho, J. M. Noh, "Hybrid of AFEN and PEN methods for multigroup diffusion nodal calculation," Trans. Am. Nuc. Soc., 73, 438 (1995).

18) E. Z. Muller, Z. J. Weiss, "Benchmarking with the multigroup diffusion high-order response matrix method," Ann. Nucl. Eng., 18, 535 (1991).

19) G. Buckel, K. Kufner, B. Stehle, "Benchmark calculations for a sodium-cooled breeder reactor by two- and three-dimen- sional diffusion methods," Nucl. Sci. Eng. 64, 75 (1977).

20) T. Takeda, G. Ikeda, "3-D neutron transport benchmarks," J. Nucl. Sci. Technol., 28, 656 (1991).

21) J. C. Lefebvre, J. Mondot, J. P. West, "Benchmark calculations of power distribution within assemblies," NEACRP-L336, OECD Nuclear Energy Agency (1991).

22) E. E. Lewis et al., "Benchmark specification for deterministic 2-D/3-D MOX fuel assembly transport calculations without spatial homogenization (C5G7MOX)," NEA/NSC/ DOC(2001)4. OECD Nuclear Energy Agency (2001).

23) J. Y. Lim, M. H. Kim, "A new LFR design concept for effective TRU transmutation," Prog. Nucl. Energy, 49, 230 (2007).

24) H. G. Joo, T. J. Downar, "An incomplete domain decomposition preconditioning method for nonlinear nodal kinetics calculations," Nucl. Sci. Eng., 123, 403 (1996). 